\section{Prevalência e padrão de distribuição do acidente vascular encefálico em idosos submetidos a tratamento fisioterapêutico no município de Jequié,BA} Prevalence and distribution pattern of cerebrovascular accident in elderly
under physical therapeutic treatment in the city of J equié, BA, Brazil

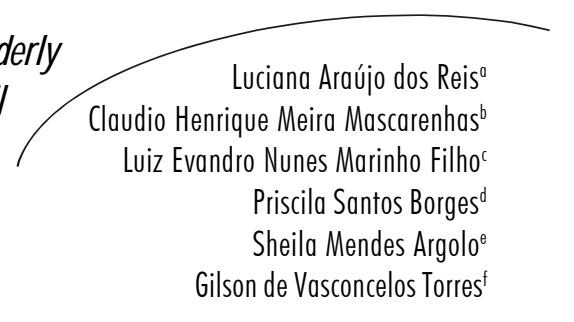

Luciana Araújo dos Reis ${ }^{0}$

\section{Resumo}

Este estudo teve por objetivo identificar a prevalência e padrão de distribuição do Acidente Vascular Encefálico (AVE) em idosos atendidos na Clínica-Escola de Fisioterapia da Universidade Estadual do Sudoeste da Bahia. Trata-se de estudo de caráter descritivo, com delineamento transversal e abordagem retrospectiva, de agosto de 2005 a agosto de 2006. Foram analisados 48 prontuários de pacientes portadores de AVE, de ambos os sexos, com idade igual ou superior a 60 anos, através da observação de 120 prontuários de pacientes atendidos no mesmo período. Com a análise dos dados, observou-se que, entre os casos de AVE, 52,1\% eram do sexo feminino; $43,75 \%$ se encontravam na faixa etária entre $60-69$ anos; 39,58\% eram aposentados; $64 \%$ das mulheres viviam sem companheiro, enquanto $73,91 \%$ dos homens viviam acompanhados. Os homens apresentaram maiores taxas de sedentarismo (69,56\%), etilismo (39,13\%) e tabagismo (39,13\%); e em ambos os sexos, os antecedentes familiares mais encontrados foram hipertensão arterial e AVE. Este estudo constatou uma alta prevalência do AVE entre os idosos, além de grande distribuição de fatores de risco e antecedentes familiares. Nessa perspectiva, faz-se necessária a criação de estratégias preventivas para a manutenção da independência e qualidade de vida dos idosos.

\author{
Palavras-chave: \\ Idoso. Acidente \\ Cerebral Vascular. \\ Estudos Transver- \\ sais. Epidemiologia \\ Descritiva. Estudos \\ Retrospectivos
}

\footnotetext{
Universidade Federal do Rio Grande do Norte

Programa de Pós-Graduação, Centro de Ciências da Saúde

Natal, RN, Brasil

Correspondência / Correspondence

Luciana Araújo dos Reis

E-mail: cianareis@hotmail.com
} 


\section{Abstract}

This study aimed to identify the prevalence and distribution pattern of Cerebrovascular Accident on elderly attended at the Clínica-Escola de Fisioterapia (Physical Therapy School Clinic) of the Universidade Estadual do Sudoeste da Bahia. It's a descriptive cross-sectional study with retrospective approach from August 2005 to August 2006. Forty-eight records of male and female patients with Cerebrovascular Accident were analyzed, aged 60 years or older, through observation of 120 records of patients attended within the same period. Through data analysis, it was observed that among the cases of Cerebrovascular Accident, $52.1 \%$ were female; $43.75 \%$ were in the age group of $60-69$ years; $39.58 \%$ were retired; $64 \%$ of women lived without a mate, while $73.91 \%$ of men lived with a mate. Men showed higher sedentarism rates (69.56\%), alcohol abuse $(39.13 \%)$ and smoking (39.13\%); and for both sexes, the most common family histories were hypertension and cerebrovascular accident. This study found a high prevalence of Cerebrovascular Accident among the elderly, besides of great distribution of risk factors and family history. Under this perspective, preventive strategies to the maintenance of the independence and quality of life of the elderly must be created.

Key words: Aged. Stroke. Cross-Sectional Studies. Epidemiology Descriptive.

Retrospective Studies

\section{INTRODUÇÃO}

O envelhecimento populacional ocorreu inicialmente em países desenvolvidos, mas nos últimos anos esse processo tem-se apresentado de forma acentuada nos países em desenvolvimento. No Brasil, o número de idosos passou de 3 milhões em 1960, para 7 milhões em 1975 e 14 milhões em 2002. ${ }^{1}$ Este crescimento é reflexo do aumento gradual da longevidade, conjuntamente com as reduções das taxas de natalidade e mortalidade. Estimativas para a população idosa brasileira apontam que, até 2020, o país terá 32 milhões de pessoas com idade superior a 60 anos. $^{2}$

O envelhecimento biológico é um fenômeno associado a profundas mudanças na atividade das células, tecidos e órgãos, e à redução da eficácia de um conjunto de processos fisiológicos, resultando em declínios progressivos na saúde do indivíduo idoso, afe- tando sua capacidade de viver com independência. ${ }^{3}$

O processo de envelhecimento em idades avançadas está associado a alterações físicas, psicológicas e sociais, bem como ao surgimento de condições patológicas advindas de hábitos de vida inadequados (tabagismo, alcoolismo, alimentação incorreta, ausência de atividade física regular, entre outros). ${ }^{2} \mathrm{O}$ aumento na longevidade tem contribuído para o surgimento de doenças crônicas, como as desordens demenciais; os cânceres; comprometimentos cerebrovasculares, a exemplo do Acidente Vascular Encefálico (AVE), que desenvolve seqüelas que acarretam prejuízos na qualidade de vida, reduzindo a capacidade para realização das atividades básicas da vida diária dos idosos. ${ }^{4}$

O AVE consiste de um quadro neurológico agudo, provocado pela obstrução vascu- 
lar que determina isquemia em determinada área encefálica ou pelo rompimento de vasos sanguíneos que irrigam essa região, levando à hemorragia. Esta patologia envolve um rápido desenvolvimento de sinais clínicos como conseqüência de distúrbios locais ou globais da função da área comprometida com duração maior que 24 horas. ${ }^{5,6}$

Anualmente, ocorrem cerca de cinco milhões de mortes e 15 milhões de AVEs que não levam a óbito, com 50 milhões de sobreviventes de AVE e Ataque Isquêmico Transitório (AIT), sendo que, destes, um em cada seis afetados será atingido por novo AVE ou AIT em cinco anos. ${ }^{7}$ Entretanto, apesar do envelhecimento da população, as taxas de mortalidade vêm diminuindo acentuadamente em muitos países. Esse fato é atribuível ao aumento da sobrevida do AVE, à diminuição da sua gravidade e ao aumento no controle dos fatores de risco. Apesar da elevada taxa de sobrevida desta patologia e do melhor prognóstico, cerca $90 \%$ dos sobreviventes desenvolvem algum tipo de deficiência, conduzindo à incapacidade, dependência funcional e, conseqüentemente, perda da qualidade de vida. ${ }^{5}$

Como prevenção primária, a principal intervenção seria diminuir ou abolir os fatores de risco para a ocorrência desta patologia. Esses fatores podem ser divididos em nãomodificáveis (idade, sexo, raça e história familiar) e modificáveis (hipertensão arterial, diabetes mellitus, tabagismo, obesidade, vida sedentária, uso de álcool, uso de anticoncepcionais, uso de drogas, enxaqueca e dislipidemias), sendo estes últimos considerados os mais importantes, pois são passíveis de inter- venção. ${ }^{7}$ Dentre os fatores modificáveis, o principal fator de risco é a hipertensão arterial (HA), pois indivíduos com pressão arterial sistólica maior que $160 \mathrm{~mm} \mathrm{Hg}$ e diastólica maior que $95 \mathrm{mmHg}$ possuem risco aumentado para o AVE de quatro vezes em relação à população em geral. ${ }^{6}$

Além de contribuir para a morbidade e mortalidade, os danos causados ao indivíduo, à sua família e à sociedade - pela limitação à realização de suas atividades de vida diária, pelos anos perdidos de produtividade e pelos elevados custos financeiros de hospitalização e reabilitação - transformam o AVE como relevante questão de saúde pública. Neste sentido, destaca-se a importância científica e social de investigar a prevalência do AVE na população de idosos, que é o grupo mais suscetível a esta patologia. ${ }^{6}$

O objetivo do presente estudo foi identificar a prevalência e o padrão de distribuição do AVE em idosos atendidos no setor de neurogeriatria da Clínica-Escola de Fisioterapia (CEF) da Universidade Estadual do Sudoeste da Bahia - Campus de Jequié.

\section{METODOLOGIA}

Trata-se de estudo de caráter descritivo, com delineamento transversal e abordagem retrospectiva de agosto de 2005 a agosto de 2006, que envolveu a coleta de dados em todos os prontuários de idosos, ou seja, indivíduos acima de 60 anos, atendidos no setor de neurogeriatria da Clínica-Escola de Fisioterapia (CEF) da UESB no período acima cita- 
do, perfazendo um total de 120 prontuários. Foi adotado como critério de inclusão apenas os idosos portadores de AVE, totalizando 48 prontuários dos 120 analisados.

No presente estudo, foi assumido o conceito de idoso preconizado pela ONU (Organização das Nações Unidas), OMS (Organização Mundial de Saúde) e utilizado no Brasil, ou seja, toda pessoa com mais de 60 anos. ${ }^{8,9}$

O Serviço de Fisioterapia da CEF/UESB foi escolhido por ser um centro de referência no município de Jequié/BA e região no tratamento fisioterapêutico de patologias neurológicas.

Como instrumento para a coleta de dados, utilizou-se uma ficha adaptada para as informações de interesse neste estudo. Os procedimentos de coleta de dados realizaramse após parecer favorável do comitê de ética da UESB, atendendo aos aspectos éticos cons- tantes na Resolução n 196/96 do Conselho Nacional de Saúde.

Os dados coletados foram analisados de maneira descritiva e posteriormente tabulados.

\section{RESULTADOS}

A análise dos prontuários revelou que 23 indivíduos $(47,91 \%)$ correspondiam ao sexo masculino e $25(52,09 \%)$ ao sexo feminino (tabela 1). As mulheres apresentaram maior percentual nas faixas etárias acima de 70 anos, enquanto os homens foram maioria nas faixas inferiores a 70 anos.

A média de idade dos indivíduos foi de 72,13 anos. O grupo que corresponde ao sexo masculino apresentou idade média de 71,78 anos, enquanto que os indivíduos do sexo feminino apresentaram uma média de idade de 72,48 anos.

Tabela 1 - Freqüência do AVE por gênero e faixa etária. Jequié, BA, 2007.

\begin{tabular}{ccccccc}
\hline \multirow{2}{*}{$\begin{array}{c}\text { Idade } \\
\text { (em décadas) }\end{array}$} & \multicolumn{3}{c}{ Sexo } & \multicolumn{2}{c}{ Total } \\
\cline { 2 - 6 } & $\mathrm{n}$ & $\%$ & $\mathrm{n}$ & $\%$ & $\mathrm{n}$ & $\%$ \\
$60-69$ & 10 & 20,84 & 11 & 22,92 & 21 & 43,75 \\
$70-79$ & 11 & 22,92 & 8 & 16,66 & 19 & 39,58 \\
$\geq 80$ & 4 & 8,33 & 4 & 8,33 & 8 & 16,67 \\
\hline Total & 25 & 52,09 & 23 & 47,91 & 48 & 100 \\
\hline
\end{tabular}


Quanto à ocupação dos indivíduos deste estudo, observou-se que 39,58\% dos idosos são aposentados; $16,67 \%$ têm trabalhos domésticos; $29,27 \%$ têm outras profissões, como comerciante, mecânico ou agricultor; e 14,58\% não citaram a profissão. Em relação ao estado civil, 64\% das mulheres vivem sem companheiro; contrapondo-se aos $73,91 \%$ dos homens que vivem com a presença de uma companheira.
$\mathrm{Na}$ análise dos hábitos de vida, observou-se que os homens apresentaram maiores taxas de sedentarismo $(69,56 \%)$, etilismo $(39,13 \%)$ e tabagismo $(39,13 \%)$ do que as mulheres (tabela 2). Entretanto, a tabela demonstra que uma alta porcentagem das mulheres não citou seus hábitos de vida, o que pode comprometer a fidedignidade desses dados.

Tabela 2 - Distribuição de hábitos de vida segundo gênero. Jequié, BA, 2007.

\begin{tabular}{|c|c|c|c|c|}
\hline \multirow{3}{*}{ Variáveis } & \multicolumn{4}{|c|}{ Sexo } \\
\hline & \multicolumn{2}{|c|}{ Feminino } & \multicolumn{2}{|c|}{ Masculino } \\
\hline & $\mathrm{n}$ & $\%$ & $\mathrm{~N}$ & $\%$ \\
\hline \multicolumn{5}{|l|}{ Sedentarismo } \\
\hline Sim & 10 & 40 & 16 & 69,56 \\
\hline Não & 0 & 0 & 3 & 13,04 \\
\hline Não cita & 15 & 60 & 4 & 17,40 \\
\hline \multicolumn{5}{|l|}{ Etilismo } \\
\hline Sim & 9 & 36 & 9 & 39,13 \\
\hline Não & 3 & 12 & 12 & 52,17 \\
\hline Não cita & 13 & 52 & 2 & 8,70 \\
\hline \multicolumn{5}{|l|}{ Tabagismo } \\
\hline Sim & 7 & 28 & 9 & 39,13 \\
\hline Não & 3 & 12 & 13 & 56,52 \\
\hline Não cita & 15 & 60 & 1 & 4,35 \\
\hline
\end{tabular}

Os dados da tabela 3 mostram que as maiores dependências dos homens são tomar banho (43,48\%), vestir-se $(39,13 \%)$ e calçar sapatos $(39,13 \%)$. Já para as mulheres, pente- ar os cabelos (52\%), tomar banho (44\%), vestir-se (40\%) e calçar os sapatos (40\%) são as atividades mais citadas como causadoras de dependência.

Tabela 3 - Classificação dos idosos quanto à capacidade funcional na realização das atividades cotidianas. Jequié/BA, 2007.

\begin{tabular}{|c|c|c|c|c|c|c|c|c|}
\hline \multirow[t]{3}{*}{ Atividades cotidianas } & \multicolumn{8}{|c|}{ Sexo } \\
\hline & \multicolumn{4}{|c|}{ Feminino } & \multicolumn{4}{|c|}{ Masculino } \\
\hline & $\mathrm{I}(\%)^{1}$ & $\mathrm{P}(\%)^{2}$ & $\mathrm{D}(\%)^{3}$ & $\mathrm{NC}(\%)^{4}$ & $\mathrm{I}(\%)$ & $\mathrm{P}(\%)$ & $\mathrm{D}(\%)$ & $\mathrm{NC}(\%)$ \\
\hline Tomar banho & 8 & 28 & 44 & 20 & 26,09 & 17,40 & 43,48 & 13,04 \\
\hline Escovar os dentes & 36 & 8 & 24 & 32 & 52,17 & 8,70 & 30,43 & 8,70 \\
\hline Pentear os cabelos & 12 & 20 & 52 & 16 & 47,83 & 4,35 & 30,43 & 17,40 \\
\hline Vestir-se & 12 & 28 & 40 & 20 & 30,43 & 17,40 & 39,13 & 13,04 \\
\hline Calçar sapatos & 12 & 20 & 40 & 28 & 34,78 & 8,70 & 39,13 & 17,40 \\
\hline Alimentação & 44 & 12 & 16 & 28 & 43,48 & 21,74 & 21,74 & 13,04 \\
\hline
\end{tabular}

${ }^{1}$ I independente ${ }^{2} \mathrm{P}$ : parcialmente dependente ${ }^{3} \mathrm{D}$ : dependente $4 \mathrm{NC}$ : não cita 
De acordo com a tabela 4, os antecedentes familiares com maior freqüência entre homens e mulheres foram a hipertensão arterial $(47,83 \%$ e $52 \%)$ e o AVE $(47,83 \%$ e $40 \%)$, respectivamente. Outros antecedentes que constituem fatores de risco significativos, neste estudo, foram o diabetes e as cardiopatias, mais freqüentes no sexo masculino.

Tabela 4 - Freqüência de antecedentes familiares de acordo com o gênero. Jequié, BA, 2007.

\begin{tabular}{ccccc}
\hline Antecedentes familiares & \multicolumn{3}{c}{ Sexo } \\
\cline { 2 - 5 } & \multicolumn{2}{c}{ Feminino } & \multicolumn{2}{c}{ Masculino } \\
\cline { 2 - 5 } Diabetes & $\mathrm{n}$ & $\%$ & $\mathrm{n}$ & 39,13 \\
\cline { 2 - 5 } Hipertensão Arterial & 6 & 24 & 9 & 47,83 \\
Cardiopatia & 13 & 52 & 71 & 30,43 \\
Neoplasia & 6 & 4 & 5 & 21,74 \\
Acidente Vascular Encefálico & 1 & 40 & 11 & 47,83 \\
Outros & 1 & 4 & 0 & 0
\end{tabular}

Tanto os indivíduos do sexo masculino quanto os do sexo feminino apresentaram como queixa principal a hemiplegia, com $34,78 \%$ e $32 \%$, respectivamente (tabela 5). Outras queixas relatadas pelas mulheres foram a presença de dor e fraqueza muscular, com $28 \%$ cada um; enquanto que entre os homens a fraqueza muscular foi a segunda queixa mais citada, com $26 \%$. Na mesma tabela, verificouse a distribuição da localização da dor segundo o gênero dos pacientes, na qual se pode identificar como principal fonte de dor para o sexo feminino os membros inferiores, com $16 \%$. No homem, essa localização abrange os membros superiores e inferiores, em que ambos apresentam 13,04\% dos acometimentos. 
Tabela 5 - Distribuição de queixa principal e localização da dor segundo o gênero. Jequié/BA, 2007.

\begin{tabular}{|c|c|c|c|c|}
\hline \multirow{3}{*}{$\frac{\text { Variáveis }}{\text { Queixa Principal }}$} & \multicolumn{4}{|c|}{ Sexo } \\
\hline & \multicolumn{2}{|c|}{ Feminino } & \multicolumn{2}{|c|}{ Masculino } \\
\hline & $\mathrm{n}$ & $\%$ & $\mathrm{n}$ & $\%$ \\
\hline Presença de dor & 7 & 28 & 5 & 21,74 \\
\hline Diminuição de ADM & 1 & 4 & 1 & 4,35 \\
\hline Deficiência na marcha & 3 & 12 & 5 & 21,74 \\
\hline Fraqueza muscular & 7 & 28 & 6 & 26,09 \\
\hline Seqüela de AVE & 3 & 12 & 5 & 21,74 \\
\hline Dormência & 1 & 4 & 3 & 13,04 \\
\hline $\begin{array}{l}\text { Ausência de movimentos unilateral } \\
\text { (hemiplegia) }\end{array}$ & 8 & 32 & 8 & 34,78 \\
\hline Diminuição da funcionalidade & 3 & 12 & 1 & 4,35 \\
\hline $\begin{array}{l}\text { Outras (úlceras, alterações de equilíbrio, } \\
\text { insuficiência urinária, edema, tonturas) }\end{array}$ & 4 & 16 & 4 & 17,40 \\
\hline Localização da dor & $\mathrm{n}$ & $\%$ & $\mathrm{n}$ & $\%$ \\
\hline MMSS (membros superiores) & 2 & 8 & 3 & 13,04 \\
\hline MMII (membros inferiores) & 4 & 16 & 3 & 13,04 \\
\hline Coluna & 2 & 8 & 1 & 4,35 \\
\hline Rosto & 1 & 4 & 0 & 0 \\
\hline
\end{tabular}

\section{DISCUSSÃO}

O Acidente Vascular Encefálico é, hoje, uma das causas mais comuns de disfunção neurológica que ocorre na população adulta e é responsável por aproximadamente $25 \%$ dos óbitos nos países desenvolvidos. Mesmo quando não é mortal, leva com freqüência à invalidez parcial ou total do indivíduo, com graves repercussões para a pessoa acometida, sua família e a sociedade. ${ }^{10}$ No presente estudo, a prevalência de idosos com AVE atendidos no setor de neurogeriatria da CEF, no período estudado, foi de $40 \%$.

Em relação à distribuição por sexo, a maioria dos pacientes era do sexo feminino (52,09\%), em contradição com estudo realizado por Carvalho e Mello. Contudo, na literatura predomina a incidência relativamente maior em homens em relação às mulheres. ${ }^{11,12}$
Os homens foram maioria entre as faixas etárias mais jovens (menor que 70 anos), enquanto as mulheres apresentaram maior percentual em faixas mais avançadas (acima de 70 anos). Este fato pode estar relacionado à maior longevidade das mulheres, que tem sido atribuída à menor exposição aos fatores de risco como tabagismo e etilismo, e à atitude das mesmas em relação às doenças e incapacidades. ${ }^{13}$ Em contrapartida, outro estudo demonstrou níveis de incidência do AVE maiores em homens de faixa etária mais avançada (acima de 75 anos). ${ }^{14}$

A idade média dos indivíduos foi de 72,13 anos, apresentando semelhança com outros estudos. ${ }^{15,16}$ Em relação à idade média por sexo, os homens apresentaram 71,78 anos; enquanto as mulheres, 72,48 anos. Em outro estudo, a média de idade entre homens e mulheres foi de 62,37 e 65,95 anos, respectivamente, 
demonstrando um aparecimento mais precoce da patologia nessa população. ${ }^{12}$

A profissão da maioria dos entrevistados, em atividades de pouca qualificação profissional ("trabalho braçal" ou de menor envolvimento intelectual), no setor de serviços de atividade informal ou doméstico, pode refletir uma escolaridade precária. Níveis baixos de educação podem contribuir para o surgimento da doença, e este quadro, combinado aos fatores socioeconômicos e culturais, pode dificultar a conscientização sobre os cuidados com a saúde do indivíduo, a aderência ao tratamento e a manutenção de um estilo de vida apropriado que limita os efeitos dos fatores de risco. ${ }^{13}$

Em determinada pesquisa, a maioria dos homens vive com a presença de um cônjuge, enquanto as mulheres vivem sem companheiro. ${ }^{12}$ Estes resultados estão em conformidade ao observado em nosso estudo, em que a maioria $(73,91 \%)$ dos homens vive com a presença de uma companheira. Como em outros países, no Brasil há um número maior de mulheres idosas do que homens, sendo que essa diferença de expectativa de vida se acentua com o aumento da idade, o que explica uma menor proporção de mulheres com companheiros em relação aos homens. ${ }^{17}$

Quanto ao hábito de fumar, os homens $(39,13 \%)$ apresentaram porcentagem mais relevante do que as mulheres $(28 \%)$. As taxas encontradas, se mostraram significativas quando comparadas com os 4,3\% de tabagistas de ambos os sexos do estudo longitudinal Saúde e Qualidade de Vida na Terceira Ida- de, realizado no Rio de Janeiro em 1998. Há evidências de que a interrupção do hábito de fumar reduz o risco de infarto cerebral, enquanto a manutenção do tabagismo leva a um risco aumentado de 1,9 vezes. ${ }^{18}$ Isto pode estar atribuído às diferenças socioeconômicas regionais, pois quanto menor o poder aquisitivo, maior a prevalência de hábitos como etilismo e tabagismo. ${ }^{18} \mathrm{O}$ etilismo já obtém valores próximos entre homens $(39,13 \%)$ e mulheres (36\%), fato que pode ser explicado pela mudança de costumes socioculturais anteriormente proibidos para as mulheres.

Dados deste estudo demonstram que a maioria dos homens $(69,56 \%)$ e $40 \%$ das mulheres são sedentários. Esta alta prevalência é preocupante, pois é conhecido que a prática regular de exercícios físicos é uma estratégia preventiva primária, eficaz para manter e melhorar o estado de saúde, reduzindo o risco de patologias freqüentes na terceira idade, como o AVE. O etilismo, tabagismo e sedentarismo são fatores de risco potenciais para o acidente vascular encefálico, podendo representar risco de recorrência da patologia nessa população. ${ }^{6}$

O histórico familiar é um indicador biológico importante de aumento da susceptibilidade para o AVE, e fatores de risco importantes como diabetes, hipertensão arterial, doença cardíaca, tabagismo e hiperlipidemia potencializam o risco de eventos cerebrovasculares. ${ }^{19}$ Entre os idosos do sexo feminino, houve $52 \%$ de história familiar de hipertensão arterial e 40\% do AVE, enquanto que nos homens, tanto a hipertensão arterial quanto o AVE obtiveram porcentagens 
semelhantes $(47,83 \%)$. Outros fatores importantes encontrados foram o diabetes mellitus $\mathrm{e}$ as cardiopatias.

A maioria dos idosos que sobrevivem a um AVE são dependentes em suas atividades básicas da vida diária, como tomar banho, vestir-se, alimentar-se, pentear-se e transferirse. ${ }^{19}$ Esse fato foi comprovado neste estudo, que mostra dependência em ambos os sexos, principalmente nas atividades como tomar banho, vestir-se e calçar os sapatos.

Lesões cerebrais focais encontradas em pacientes com AVE podem produzir uma série de déficits neurológicos, a depender da área e da extensão da lesão, tais como: hemiplegia, afasia, perda hemissensorial, hemianopsia, alterações de equilíbrio, entre outros. ${ }^{6,19}$ Essas alterações afetam a capacidade funcional, muitas vezes predispondo o paciente à dependência nas atividades da vida diária. Dentre as seqüelas do AVE encontradas nos indivíduos deste estudo; a hemiplegia, a presença de dor e a fraqueza muscular foram as queixas mais citadas por ambos os sexos como causadoras de dependência para realização das atividades básicas da vida diária.

Um dado importante a ser considerado foi a ausência de informações acerca do tipo, da localização e da extensão do AVE, que proporcionariam maiores detalhes para a pesquisa.

\section{CONCLUSÃO}

Neste estudo, evidenciamos uma prevalência do AVE atingindo $40 \%$ dos idosos atendidos na CEF da UESB. O quadro patológico, independentemente do gênero, é causa de insatisfação com a vida e de limitações funcionais diversas, pela perda da autonomia decorrente das incapacidades e pelas repercussões geradas na família e na sociedade. Tendo em vista sua alta prevalência, o risco de óbito, as seqüelas e complicações dele decorrentes, assim como os altos custos para o sistema de saúde relacionados ao processo de hospitalização e reabilitação, faz-se necessário a realização de novos estudos sobre o AVE na terceira idade, visando à implementação de ações que minimizem o impacto dessa patologia na sobrevida desses indivíduos, melhorando assim sua qualidade de vida.

É ainda desejável que sejam considerados, ao se planejarem tais ações, a condição de gênero e os comportamentos preventivos e de cuidados com a saúde, diferentes, assumidos por cada sexo.

\section{NOTAS}

a Fisioterapeuta, Especialista em Saúde Pública, Docente do Departamento de Saúde da Universidade Estadual do Sudoeste da Bahia (UESB), Mestranda do Programa de Pós-Graduação em Ciências da Saúde/CCS-UFRN. E-mail: cianareis@hotmail.com

b Fisioterapeuta, Especialista em Saúde Pública, Docente do Departamento de Saúde da UESB. E-mail: claudio12fisio@hotmail.com

c,de Acadêmicos do curso de graduação em Fisioterapia da UESB. E-mails: luizevandromarinho@hotmail.com; piuzinhaborges@hotmail.com; sheu_mendes@hotmail.com

f Enfermeiro, Doutor em Enfermagem/EERP-USP, Docente do Programa de Pós-Graduação em Ciências da Saúde/CCS, Universidade Federal do Rio Grande do Norte, orientador da pesquisa. E-mail: gvt@ufrnet.br. 


\section{REFERÊNCIAS}

1. Veras R. Em busca de uma assistência adequada à saúde do idoso: revisão da literatura e aplicação de um instrumento de detecção precoce e de previsibilidade de agravos. Cad. Saúde Pública 2003 jun; 19(3): 72-80.

2. Tribess S, Virtuoso Junior JS. Prescrição de exercícios físicos para idosos. Revista sauìde.com 2005; 1 (2):163-72.

3. Rebelatto JR, Calvo JI, Orejuela JR, et al. Influência de um programa de atividade física de longa duração sobre a força muscular manual e a flexibilidade corporal de mulheres idosas. Revista brasileira de fisioterapia 2006; 10(1): 61-9.

4. Souza NR, Oliveira AA, Oliveira MML, et al. Olhar sobre o cuidador de idosos dependentes. Revista sauìde.com 2005; 1(1): 51-9.

5. Makiyama TY, Battisttella LR, Litvoc J, et al. Estudo sobre a qualidade de vida de pacientes hemiplégicos por acidente vascular cerebral e de seus cuidadores. Acta fisiaitrica 2004; 11(3): 106-9.

6. Virginia C, Passos D, Reis LA. Fatores de risco para o Acidente Vascular Encefálico. [monografia]. Jequié: Universidade Estadual do Sudoeste da Bahia - UESB/BA, 2006.

7. Silva F. Acidente vascular cerebral isquêmico Prevenção: Aspectos atuais - É preciso agir. Medicina Interna 2004; 11(2): 104-11.

8. Coutinho ESF, Silva SD. Uso de medicamentos como fator de risco para fratura grave decorrente de queda em idosos. Cad Saúde Publica 2002 set./out.; 18(5): 132-6.

9. Pizzolli SML, Ruete REN, Gagel CA, Santos MD. Avaliação gerontologica através do índice de Kartz (nível de dependecia) no Lar de idosos de Paragaçu Paulista. Omnia Saúde 2006; 1(1): 26-31.

10. Costa AM, Duarte E. Atividade física e a relação com a qualidade de vida, de pessoas com seqüelas de acidente vascular cerebral isquêmico. Revista brasileira de ciência \& movimento 2002 jan; 10(1): 47-54.
11. Umphred, D. A. Reabilitação Neurológica. 4.ed. Barueri: Manole; 2004. p. 782-833.

12. Marques S, Rodrigues RAP, Kusumota L. Cerebrovascular accident in the aged: changes in family relations. Rev Lat Am Enfermagem 2006 maio/jun; 14 (3): 37-45.

13. Coelho Filho JM, Ramos LR. Epidemiologia do envelhecimento no Nordeste do Brasil: resultados de inquérito domiciliar. Rev Saúde Pública 1999 out; 33(5): 21-5.

14. Lebrão ML, Laurenti R. Condições de saúde. In: Lebrão ML, Duarte YAO, (organizadores). SABE - Saúde, Bem-estar e Envelhecimento - O projeto Sabe no município de São Paulo: uma abordagem inicial. Brasília (DF): Organização PanAmericana da Saúde, 2003. p.75-89.

15. Feigin VL, Lawes CM, Bennett DA, et al. Stroke epidemiology: a review of population-based studies of incidence, prevalence, and case-fatality in the late 20 th century. Lancet Neurol 2003 jan; 2(1): 43-53.

16. Garrido R, Menezes PR. O Brasil está envelhecendo: boas e más notícias por uma perspectiva epidemiológica. Rev Bras Psiquiatr 2002 abr; 24: 11-9.

17. Anderson MIP, Assis M, Pacheco LC, et al. Saúde e qualidade de vida na terceira idade. Textos Envelhecimento 1998 nov; 1(1): 83-9.

18. Carvalho e Mello ALL. Prevalência e sobrevida de casos de acidente vascular encefálico, no município do Rio de Janeiro, no ano de 1998. [acesso em: 2008 jan 21] Disponível em: URL: http://teses.cict.fiocruz.br/pdf/latinialm.pdf Rio de Janeiro, 2003.

19. Delisa JA. Tratado de Medicina de Reabilitação: princípios e prática. 3.ed. São Paulo: Manole; 2002. v. 2.

Recebido em: 25/5/2007

Reapresentado: 29/8/2007

Aprovado: 10/12/2007 